\title{
Targeting both Notch and ErbB-2 signalling pathways is required for prevention of ErbB-2-positive breast tumour recurrence
}

\author{
K Pandya', K Meeke ${ }^{2}$, AG Clementz ${ }^{3}$, A Rogowski', J Roberts ${ }^{2}$, L Miele ${ }^{4}$, KS Albain ${ }^{5}$ and C Osipo*,1,2,3,6,7 \\ 'Molecular Biology Program, Loyola University Chicago, 2160 South First Avenue, Maywood, IL 60153, USA; ${ }^{2}$ Oncology Institute, Stritch School of \\ Medicine at Loyola University Medical Center, 2160 South First Avenue, Maywood, IL 60 I 53, USA; ${ }^{3}$ Molecular and Cellular Biochemistry Program, Loyola \\ University Chicago, 2160 South First Avenue, Maywood, IL 60153, USA; ${ }^{4}$ University of Mississippi Cancer Institute, 350 Woodrow Wilson Drive, Suite \\ 600, Jackson, MS 39213, USA; ${ }^{5}$ Department of Medicine, Loyola University Chicago, 2160 South First Avenue, Maywood, IL 60153, USA; ${ }^{6}$ Department \\ of Pathology, 2160 South First Avenue, Maywood, IL 60153, USA; 'Department of Microbiology and Immunology, 2160 South First Avenue, Maywood, \\ IL 60153, USA
}

BACKGROUND: We reported that Notch-I, a potent breast oncogene, is activated in response to trastuzumab and contributes to trastuzumab resistance in vitro. We sought to determine the preclinical benefit of combining a Notch inhibitor ( $\gamma$-secretase inhibitor (GSI)) and trastuzumab in both trastuzumab-sensitive and trastuzumab-resistant, ErbB-2-positive, BT474 breast tumours in vivo. We also studied if the combination therapy of lapatinib plus GSI can induce tumour regression of ErbB-2-positive breast cancer. METHODS: We generated orthotopic breast tumour xenografts from trastuzumab- or lapatinib-sensitive and trastuzumab-resistant BT474 cells. We investigated the antitumour activities of two distinct GSIs, LY 4II 575 and MRK-003, in vivo.

RESULTS: Our findings showed that combining trastuzumab plus a GSI completely prevented (MRK-003 GSI) or significantly reduced (LY 4I I 575 GSI) breast tumour recurrence post-trastuzumab treatment in sensitive tumours. Moreover, combining lapatinib plus MRK-003 GSI showed significant reduction of tumour growth. Furthermore, a GSI partially reversed trastuzumab resistance in resistant tumours.

CONCLUSION: Our data suggest that a combined inhibition of Notch and ErbB-2 signalling pathways could decrease recurrence rates for ErbB-2-positive breast tumours and may be beneficial in the treatment of recurrent trastuzumab-resistant disease.

British Journal of Cancer (201 I) I 05, 796-806. doi:I0.1038/bjc.20II.32I www.bjcancer.com

Published online 16 August 2011

(c) 201 I Cancer Research UK

Keywords: ErbB-2; trastuzumab; Notch- I; GSI; recurrence; resistance

We have recently shown that the epidermal growth factor receptor-2 (ErbB-2 or HER-2/neu) inhibits Notch-1 activity (Osipo et al, 2008). The ErbB-2 is a receptor tyrosine kinase that belongs to the epidermal growth factor receptor family. It is activated by growth factor-independent homodimerisation or growth factor-dependent heterodimerisation with family members, epidermal growth factor receptor (EGFR), ErbB-3, or ErbB-4 (Yarden, 2001). The ErbB-2 transmits its signal inside the cell to stimulate proliferation and survival (Yarden, 2001; Yarden and Sliwkowski, 2001). The ErbB-2 proto-oncogene is amplified, overexpressed, and/or hyperactive in $15-25 \%$ of patients with breast cancers, such as the 'HER-2 positive subtype', which is oestrogen receptor $\alpha(\mathrm{ER} \alpha)$ and progesterone receptor (PR) negative, or a subset of the 'luminal B subtype', which is ER $\alpha / \mathrm{PR}$ positive (Slamon et al, 1989; Menendez et al, 2006). The clinical importance of targeted therapy based on increased expression and activity of ErbB-2 is underestimated if just based on the $\mathrm{ER} \alpha / \mathrm{PR}$ classification. This is because it has also been shown that the luminal A $(\mathrm{ER} \alpha / \mathrm{PR}+$, HER-2-) subtype of breast tumours, which represents $\sim 70 \%$ of breast cancers, might, after becoming resistant to antihormonal

*Correspondence: Dr C Osipo; E-mail: cosipo@lumc.edu

Received 9 May 201 I; revised 12 July 2011; accepted I8 July 201 I; published online 16 August 201 I therapy such as tamoxifen (Benz et al, 1992; Osipo et al, 2003, 2005a) or aromatase inhibitors (Brodie et al, 2009), evolve to overexpress ErbB-2. Some luminal B and the HER-2 + subtypes of breast cancer, which contain gene amplification of ErbB-2, are associated with poor prognosis, aggressive disease, and resistance to some cytotoxic (Yokoyama et al, 2005) and endocrine therapies (Shou et al, 2004). Furthermore, the increased ErbB-2 activity in the luminal A tumours that become resistant to antihormonal therapy may be an important target for alternative or combination therapy that attempts to prevent and/or reverse the resistance to antihormonal therapy.

Trastuzumab is a humanised monoclonal antibody that binds the extracellular, juxtamembrane domain of ErbB-2 (Carter et al, 1992). Trastuzumab blocks ErbB-2 at the cell surface and inhibits receptor-receptor interactions, thereby slowing growth by inhibiting activation and signalling (Sliwkowski et al, 1999; Baselga et al, 2001; Nagata et al, 2004; Nahta and Esteva, 2006). The best efficacy and positive therapeutic outcome with trastuzumab is observed in women with tumours that overexpress, have an amplification, or have high activity of ErbB-2 (Muss et al, 1994; Nabholtz and Slamon, 2001; Slamon and Pegram, 2001; Menendez et al, 2006). Although trastuzumab has had a tremendous impact on improving survival for women with ErbB-2-positive breast cancer, resistance to trastuzumab remains a serious and 
unacceptable clinical problem, particularly in women with metastatic breast cancer. Unfortunately, when given as a single agent, $66-88 \%$ of women with metastatic breast cancer are resistant to trastuzumab (Cobleigh et al, 1999; Vogel et al, 2002). Furthermore, some women with metastatic breast cancer, who initially respond to trastuzumab-based treatments that include chemotherapy, develop resistance within the first year (Amar et al, 2008). Approximately $15 \%$ of women with non-metastatic breast tumours who receive trastuzumab in the adjuvant setting will develop recurrent breast cancer. Recently, lapatinib, a smallmolecule, dual EGFR/ErbB-2 tyrosine kinase inhibitor (TKI), has been effective in women with trastuzumab-resistant disease when combined with a cytotoxic agent, the selective oral fluoropyrimidine capecitabine that is ultimately converted to 5-fluorouracil (Nahta et al, 2007). However, lapatinib resistance has been observed in cell culture models (Xia et al, 2006) and, clinically, patients treated with lapatinib become resistant within the first year of treatment (Li et al, 2008; Widakowich et al, 2008; Kaufman et al, 2009; Lacerda et al, 2010). Thus, despite initial efficacy in the treatment of metastatic disease with agents that target the ErbB receptor pathways, resistance occurs with few clinical means currently available to circumvent it (Li et al, 2008).

We have identified Notch-1 as a novel target in trastuzumabresistant breast cancer (Osipo et al, 2008). Notch-1 is a type I membrane receptor that is a breast oncogene (Dievart et al, 1999) and a potent cell-fate modulator (Politi et al, 2004). Increased coexpression of Notch-1 and its ligand Jagged-1 predicts the poorest overall survival in women with breast cancer (Reedijk et al, 2005; Dickson et al, 2007). Furthermore, Notch-1 mRNA was found to be elevated in breast cancer cells resistant to gefitinib, an EGFR TKI (Piechocki et al, 2007). There are four mammalian Notch receptors (Notch-1, -2, -3, and -4) with five canonical ligands (Delta-like 1, 3, and 4 (Bettenhausen et al, 1995; Dunwoodie et al, 1997; Shutter et al, 2000) and Jagged-1 and -2 (Lindsell et al, 1995)). Both Notch ligands and receptors are type I membrane proteins, and Notch receptor-ligand interactions regulate cell fate and have been shown to be important for survival of breast tumour-initiating cells (Magnifico et al, 2009; Mine et al, 2009; Harrison et al, 2010). Notch receptors have two subunits, an extracellular Notch $\left(\mathrm{N}^{\mathrm{EC}}\right)$ and a transmembrane Notch $\left(\mathrm{N}^{\mathrm{TM}}\right)$ (Blaumueller et al, 1997; Logeat et al, 1998). Dissociation of $\mathrm{N}^{\mathrm{EC}}$ and $\mathrm{N}^{\mathrm{TM}}$ subunits is initiated upon ligand binding, which triggers two proteolytic cleavages of $\mathrm{N}^{\mathrm{TM}}$. The final cleavage within the Notch transmembrane domain is catalysed by $\gamma$-secretase, which releases the C-terminal, intracellular portion of the Notch receptor (NIC). This final step can be pharmacologically inhibited by $\gamma$-secretase inhibitors (GSIs), which prevent the release of NIC, thus inhibiting Notch-mediated transcription and cell growth. The GSIs are currently in clinical trials for the treatment of breast cancer and other solid tumours (Pannuti et al, 2010). The NIC modulates transcription via the CSL (CBF-1) transcription factor in the nucleus. Notch signalling contributes to breast cancer tumourigenesis by inhibiting differentiation, promoting survival, and/or accelerating proliferation. Here, we used three preclinical xenograft models in vivo, trastuzumab-sensitive, trastuzumabresistant, and lapatinib-sensitive xenografts, to investigate the anti-tumour benefit of combining trastuzumab or lapatinib plus a GSI on the growth of ErbB-2-positive breast tumours.

\section{MATERIALS AND METHODS}

\section{Development of BT474 trastuzumab-sensitive, trastuzumab-resistant, and lapatinib-sensitive xenografts}

BT474 cells were purchased from the American Tissue Cell Culture (ATCC, Manassas, VA, USA) and maintained as described previously (Osipo et al, 2008). BT474 breast cancer cells that are sensitive or resistant to trastuzumab as described previously
(Osipo et al, 2008) and sensitive to lapatinib were used to generate breast tumour xenografts. Five million cells were injected into two mammary fat pads of ovariectomised, FoxN1 ${ }^{\text {nu/nu }}$ athymic nude mice (Harlan Sprague-Dawley, Madison, WI, USA) followed by implantation of a $17 \beta$-estradiol-containing silastic capsule of $0.3 \mathrm{~cm}$ in length with a constant release providing $83-100 \mathrm{pg} \mathrm{ml}^{-1}$ as described previously (O'Regan et al, 1998). Each mouse was tagged on one ear to identify the specific mouse and tumour. Once tumours grew to a mean cross-sectional area (CRA) of $0.20-$ $0.30 \mathrm{~cm}^{2}$, mice were killed; tumours were extracted and retransplanted into a set of 56 mice as previously described (Osipo et al, 2005b). Tumours were allowed to grow to a mean CRA of $0.20-0.30 \mathrm{~cm}^{2}$ and mice were randomised to four treatment groups with 14 mice per group: vehicle control (sterile PBS and $2 \%$ carboxymethylcellulose), trastuzumab $\left(10 \mathrm{mg} \mathrm{kg}^{-1}\right.$ in a total volume of $100 \mu \mathrm{l}$ sterile PBS, i.p. once a week) or lapatinib (Selleck Chemical, Houston, TX, USA; $100 \mathrm{mg} \mathrm{kg}^{-1}$; fed by oral gavage, twice daily for 5 days), LY 411575 GSI (kindly provided by Drs Abdul Fauq and Todd Golde from the Mayo Clinic in Jacksonville, FL, USA; $5 \mathrm{mg} \mathrm{kg}^{-1}$; 3 days on, 4 days off) or MRK-003 (kindly provided by Merck \& Co., Whitehouse Station, NJ, USA; $100 \mathrm{mg} \mathrm{kg}^{-1}$ dissolved in $2 \%$ carboxymethylcellulose, $200 \mu \mathrm{l}$ fed by oral gavage; 3 days on, 4 days off), or trastuzumab plus MRK-003 or LY 411575 GSI or lapatinib plus MRK-003 GSI. Tumour area $(\mathrm{l} \times \mathrm{w})$ was measured weekly using Vernier calipers and cross-sectional area $((1 \times w) \Pi) / 4)$ was calculated and graphed. Tumour recurrence was monitored and tumour area measured after treatments with trastuzumab or trastuzumab plus GSI were ceased at the time tumours were no longer detectable by the Vevo 770 Ultrasound Imaging system (Visual Sonics, Toronto, Canada). Tumour recurrence post treatment was monitored up to $\sim 100$ days. Protocols that were used to study breast tumour xenografts in mice were approved by Loyola University's Institutional Animal Care and Use Committee.

\section{Immunohistochemistry}

Tumour sections $(4 \mu \mathrm{m})$ were sliced from formalin-fixed, paraffinembedded tumour samples, followed by antigen retrieval to unmask the epitope. Immunohistochemical staining was performed as described previously (Yao et al, 2010) using an antibody against the proliferation marker, Ki67 (proliferation marker, $1: 100$, DAKO, Carpinteria, CA, USA), for all four treatment groups. Biotinylated anti-mouse secondary antibody (VECTASTAIN Elite ABC Kit, Vector Laboratories, Burlingame, CA, USA) was applied to the slides to detect the primary antibody, followed by incubation with the avidin-horseradish peroxidase complex reagent (VECTASTAIN Elite ABC kit, Vector Laboratories). The staining was developed in the diaminobenzidine chromogen substrate solution (Peroxidase Substrate Kit, Vector Laboratories). Mayer's haematoxylin was used as a counterstain. Immunohistochemical analysis of Ki67 was performed using at least 3-5 tumours per treatment group. The number of Ki67-positive cells as shown on the $y$ axis was determined by taking an average number of Ki67-positive cells from 60 high-power fields (HPFs) at $\times 40$ magnification per treatment group. Haematoxylin and eosin $(\mathrm{H} / \mathrm{E})$ was performed to ensure the presence of tumour cells in the tumour sections.

\section{TUNEL assay}

Terminal deoxynucleotidyl transferase-mediated dUTP nick end labelling (TUNEL) assay with the TMR detection kit (Roche Diagnostic, Indianapolis, IN, USA) was used to detect $3^{\prime} \mathrm{OH}-$ associated DNA fragmentation resulting from apoptosis. Paraffinembedded BT474 trastuzumab- and lapatinib-sensitive tumours extracted from xenografts as described above were examined for the presence of TUNEL-positive cells from each treatment group 
according to the manufacturer's instructions. The TUNEL assay was performed using 3-5 tumours from each treatment group. The number of TUNEL-positive cells shown on the $y$ axis was the average number of TUNEL-positive cells counted per $20 \mathrm{HPFs}$ per tumour for a total of $60 \mathrm{HPFs}$ at $\times 40$ magnification per treatment group.

\section{Western blot analysis}

Frozen tumour samples from each treatment group were homogenised by grinding in liquid nitrogen and lysed in lysis buffer (50 mM HEPES, $1 \%$ Triton X-100, $150 \mathrm{~mm} \mathrm{NaCl,} 5 \mathrm{~mm}$ EDTA, $10 \mu \mathrm{g} \mathrm{ml}^{-1}$ pepstatin A, $10 \mu \mathrm{g} \mathrm{ml}^{-1}$ leupeptin, $10 \mu \mathrm{g} \mathrm{ml}^{-1}$ aprotinin, $25 \mu \mathrm{g} \mathrm{ml}^{-1}$ PMSF, $10 \mu \mathrm{g} \mathrm{ml}^{-1}$ TLCK, $10 \mu \mathrm{g} \mathrm{ml}^{-1}$ TPCK, $1 \mathrm{mM}$ o-vanadate, and $10 \mathrm{~mm} \mathrm{NaF}$ ). The lysate was sonicated, followed by centrifugation at $10000 \mathrm{~g}$ for $5 \mathrm{~min}$. The supernatant was collected and protein concentration was measured using the BCA protein assay (Thermo Fisher Scientific, Inc., Waltham, MA, USA). Tumour lysates were then subjected to SDS-PAGE and western blotting (Osipo et al, 2008) was performed using the following antibodies: phosphorylated tyrosine (Y1248)-ErbB-2 (1:1000, Millipore, Billerica, MA, USA), total ErbB-2 (1:500, AB-17, Thermo Fisher Scientific), phosphorylated-ERK1/2 (1:1000, Cell Signaling Technology, Danvers, MA, USA), total ERK1/2 (1:1000, Cell Signaling Technology), phosphorylated-AKT1 (1:1000, Cell Signaling Technology), total AKT1 (1:300, Santa Cruz Biotechnology, Santa Cruz, CA, USA), PTEN $(1: 1000$, Cell Signaling Technology), and Actin as a loading control $(1: 3000$, Sigma-Aldrich, St Louis, MO, USA). Anti-mouse $(1: 3000$, Santa Cruz Biotechnology) or anti-rabbit $(1: 2000$, Santa Cruz Biotechnology) secondary antibodies conjugated to horseradish peroxidase were used to detect the primary antibody. The blot was then developed using SuperSignal West Dura Luminol/Enhancer Solution (Thermo Fisher Scientific, Inc.) and the stained bands were visualised using the Fujifilm LAS-3000 imager (Valhalla, NY, USA).

\section{Reverse transcription real-time PCR}

Total RNA was extracted from frozen tumour samples using the RiboPure Kit (Ambion, Inc., Austin, TX, USA) according to the manufacturer's instructions. Total RNA was reverse transcribed to total cDNA using the TaqMan reverse transcription Kit (Applied Biosystems, Foster City, CA, USA) according to the manufacturer's instructions. Real-time PCR was performed using cDNA as template to detect relative expression levels of Notch targets such as HEY1 (forward primer: 5'-AGCTCCTCGGACAGCGAGCTG-3', reverse primer: $5^{\prime}$-TACCAGCCTTCTCAGCTCAGACA- $3^{\prime}$ ) and Deltex 1 (forward primer: 5'-CAGTTTCGCCAGGACACAG-3', reverse primer: $5^{\prime}$-GCAGATGTCCATATCGTAGGC-3'). The expression level of $18 \mathrm{~S}$ (forward primer: $5^{\prime}$-ATGAACCAGGTTATGACCTTGAT- ${ }^{\prime}$, reverse primer: $5^{\prime}$-CCTGTTGACTGGTCATTACA-ATA- $3^{\prime}$ ) was used as a loading control. The PCR was performed as previously described (Osipo et al, 2008).

\section{Statistical analysis}

Karyn Richlyk, a statistician at Loyola University Medical Center, assisted in determining the number of mice needed for the in vivo study. Based on experience, we hypothesised the following average tumour size for the four groups in trastuzumab- or lapatinibsensitive xenograft studies at the end of the experiment (all measurements are in cross-sectional area $\left.=\mathrm{cm}^{2}\right): 1$ vehicle $=2.0$ $($ s.d. $=0.3) ; 2$ trastuzumab or lapatinib $=0.4 \quad($ s.d. $=0.1) ; 3$ $\mathrm{GSI}=1.5$ (s.d. $=0.1$ ); and $4 \mathrm{GSI}+$ trastuzumab or lapatinib $<0.1$ $(\mathrm{s} . d .=0.01)$. For the trastuzumab-resistant xenograft study, the average tumour size for vehicle, GSI, and GSI + trastuzumab should remain the same as above. However, as these are trastuzumab-resistant tumours, we would expect the average tumour size for the trastuzumab group as $1.5 \mathrm{~cm}^{2}$. Calculations were conducted using PASS 2002 software (Kaysville, UT, USA, 2002). In a one-way ANOVA, same sample sizes of 7 were obtained for all the four groups whose means are to be compared, assuming $100 \%$ tumour take. The total sample of 28 mice achieves $95 \%$ power to detect differences among the means $v s$ the alternative of equal means using an F-test at a significance level of 0.05 . The common s.d. within a group is assumed to be between 1 and 0.01 . However, experience suggests that tumour take will be $50-70 \%$; therefore, in order to maximise the likelihood that 7 subjects per group will present with tumours, we must assume that a sample of 7 represents $50-70 \%$ from a group of 14 mice, for a total of 56 mice per experiment of four groups. Each mouse was identifiable with a numbered tag. Each tumour area on the left flank and right flank of the mouse was measured weekly with Vernier calipers. At the end of the study, tumour CRA was calculated and linear regression analysis was performed to determine the slope of the line for determination of the rate of growth for each tumour. Slopes of lines were used only if the correlation coefficients were $\geqslant 0.85$. A one-way ANOVA with Bonferroni correction for multiple comparisons and $\alpha=0.05$ was used to test statistical significance between groups for tumour growth rates, mRNA expression levels, and IHC assays. A nonpaired Student's $t$-test was used to test statistical significance between two groups.

\section{RESULTS}

\section{Anti-tumour activity of trastuzumab, GSIs, or combinations in ErbB-2-positive breast tumours in vivo}

We used several cell lines in our in vitro studies (Osipo et al, 2008); however, we chose to use BT474 ErbB-2-positive breast cancer cells as they are sensitive to trastuzumab and have been used numerous times in preclinical studies (Nagata et al, 2004; Rimawi et al, 2011) to represent the clinical setting. Osipo et al (2008) showed that ErbB-2 overexpression suppresses Notch-1 activity; thus, BT474 cells, which contain a gene amplification and therefore overexpress ErbB-2, exhibit minimal Notch-1 activity. Conversely, trastuzumab treatment increases Notch-1 transcriptional activity five-fold, and this effect was abrogated by using a GSI (Osipo et al, 2008). Breast tumour xenografts were generated using BT474 trastuzumabsensitive cells in athymic nude mice. Growth of tumours was measured in response to vehicle, trastuzumab, LY 411575 or MRK-003 GSI, or trastuzumab plus GSI. The results from two independent studies showed that trastuzumab treatment almost completely inhibited the tumour growth of BT474 breast tumour xenografts with $\sim 90-100 \%$ of tumours regressing to undetectable levels (Figure 1A and B). GSI treatment of tumours, either alone using LY 411575 (Figure 1A) or MRK-003 (Figure 1B) or in combination with trastuzumab, had no statistically significant effect on tumour growth during the treatment phase of the study compared with vehicle control or trastuzumab alone, respectively.

There is significant evidence of enhanced Notch signalling in tumour-initiating or putative breast cancer stem cells (Grudzien et al, 2010; Harrison et al, 2010; Pannuti et al, 2010). As these cells are suggested to be responsible for tumour recurrence, we discontinued treatment and measured tumour recurrence in mice where tumours were no longer detectable using the Vevo 770 Ultrasound Imager. During the recurrence phase of the study, we detected significant tumour re-growth for the previously trastuzumab-treated mice only (Figure $1 \mathrm{~A}$ and $\mathrm{B}$ ). In contrast, mice previously treated with the combination of trastuzumab plus LY 411575 GSI did not display tumour re-growth until week 25, and their tumours were significantly smaller $\left(0.12 v s 0.87 \mathrm{~cm}^{2}\right.$ for the trastuzumab-alone group, $P<0.001$; Figure 1A). Furthermore, no recurrent tumours were detectable in mice previously treated with the combination of trastuzumab plus MRK-003 GSI $(P<0.0001$; Figure 1B). Figure 2 is a Kaplan-Meier analysis of log-rank 
A
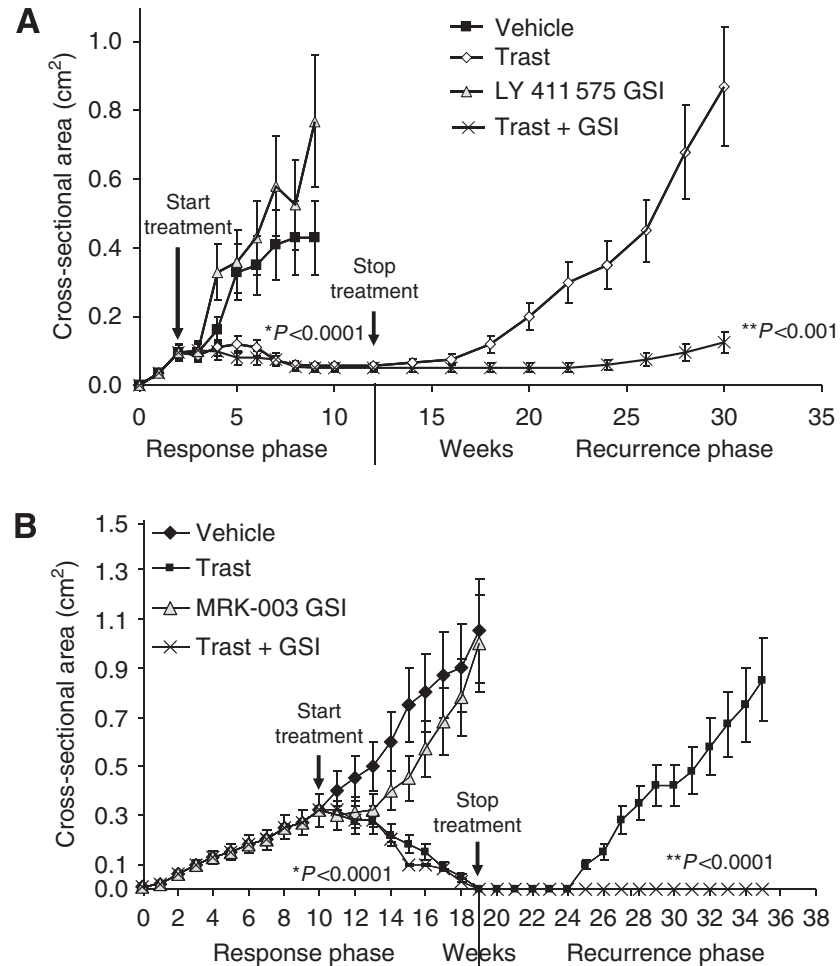

Figure I Trastuzumab (Trast) plus a $\gamma$-secretase inhibitor (GSI) prevents or reduces tumour recurrence. ErbB-2-overexpressing BT474 xenografts were generated in 56 ovariectomised, athymic nude mice by injecting $5 \times 10^{6}$ cells into both mammary fat pads. Once tumours reached a mean tumour crosssectional area of $0.20 \mathrm{~cm}^{2}$, mice were randomised and treated with vehicle $(\mid 00 \mu \mathrm{l}$ sterile PBS injected i.p. I day per week and $200 \mu \mathrm{l} 2 \%$ carboxymethylcellulose), $10 \mathrm{mg} \mathrm{kg}^{-1}$ trastuzumab in $100 \mu \mathrm{l}$ PBS injected i.p. once weekly, $5 \mathrm{mg} \mathrm{kg}^{-1}$ LY 4 II $575 \mathrm{GSI}$ (A) or $100 \mathrm{mg} \mathrm{kg}^{-1}$ MRK-003 GSI (B) in $200 \mu \mathrm{l} 2 \%$ carboxymethylcellulose, fed by oral gavage, three days on, 4 days off, or trastuzumab plus LY 4 II 575 GSI or MRK-003 GSI. Tumour area (length $\times$ width) was measured weekly using Vernier calipers. The measurements were performed up to 12 or 19 weeks. The treatments were stopped and tumour recurrence was measured up to an additional 105 days or 98 days in mice that specifically showed complete tumour regression $(\mathbf{A}$ and $\mathbf{B})$. Results from (A and $\mathbf{B})$ show mean tumour cross-sectional area $(($ area $\times \Pi) / 4)$ on the $y$ axis and time in weeks on the $x$ axis. Error bars are s.d. of the mean for 12 mice bearing tumours in the response phase of the study and 8 mice for the recurrent phase of the study. The results from $(\mathbf{A}$ and $\mathbf{B})$ also demonstrate mice bearing recurrent tumours on the $y$ axis and treatments on the $x$ axis. *Statistically significant differences between mean slopes of the curve for trastuzumab plus GSI vs GSI alone. ***statistically significant differences between mean slopes of the curve for trastuzumab vs trastuzumab plus GSI in recurrent tumours. Linear regression analyses were performed for tumour growth curves in $(\mathbf{A}$ and $\mathbf{B})$.

(Mantel-Cox) test for the rate of tumour recurrence post treatment. In both studies, $\sim 40 \%$ of mice previously treated with trastuzumab alone displayed tumour recurrence at 40 weeks. In contrast, only $10 \%$ of mice displayed tumour recurrence in the trastuzumab plus LY 411575 GSI group, and $0 \%$ of mice displayed tumour recurrence in the trastuzumab plus MRK-003 group (Figure 2). These data suggest that the main benefit of using a GSI (MRK-003) in trastuzumab-sensitive, ErbB-2-positive breast tumours is prevention of tumour recurrence. All mice were killed at the end of 40 weeks and it was confirmed via surgery that tumours were completely absent in the trastuzumab plus MRK-003 group.

\section{Expression of Notch gene targets: HEY1 and Deltex1}

Real-time RT-PCR was performed to detect canonical Notch target gene transcripts that include HEY1 and Deltex1 (Kopan and

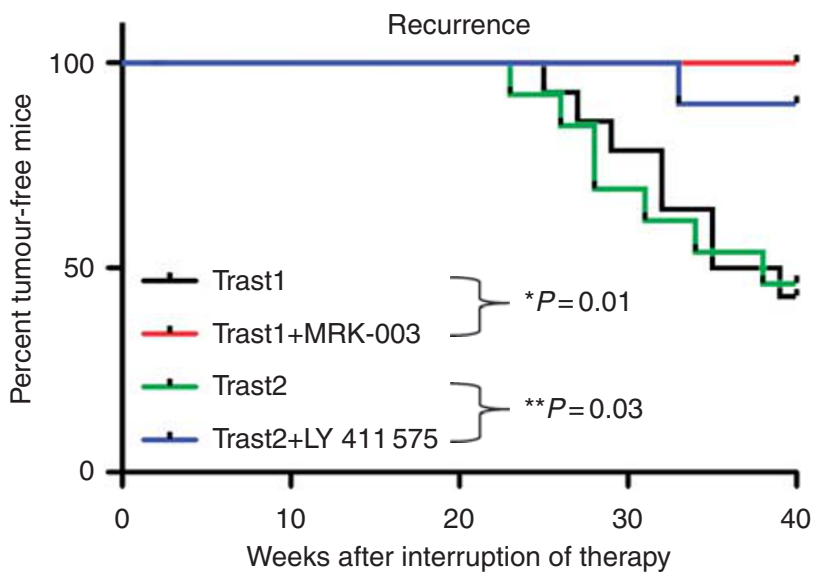

Figure 2 Kaplan-Meier curve of the percentage of tumour-free mice among Trastl, TrastI + MRK-003 GSI, Trast2, and Trast2 + LY 4II 575 GSI treatment groups using a log rank (Mantel-Cox) test. *Statistically significant differences between Trastl and Trastl + MRK-003 GSI. **Statistically significant differences between Trast2 and Trast2 + LY 4l I 575 GSI.

Ilagan, 2009) as measures of Notch signalling and efficacy of the LY 411575 (Figure 3A) or MRK-003 GSI (Figure 3B) on the Notch pathway. Figure $3 \mathrm{~A}$ and B demonstrate that LY 411575 or MRK003 GSI alone significantly inhibited HEY1 and Deltex 1 transcripts compared with vehicle control. Trastuzumab treatment alone significantly increased HEY1 by $2-4$-fold and Deltex 1 by 20 -fold compared with vehicle control (Figure 3A and B). LY 411575 or MRK-003 GSI significantly decreased the trastuzumab-induced increase in HEY1 and Deltex1 (Figure 3A and B). In addition, recurrent tumours that grew post-trastuzumab treatment showed increased baseline expression of HEY1 and Deltex 1 transcripts when compared with trastuzumab-treated tumours (Figure $3 \mathrm{~A}$ ). In contrast, recurrent tumours post-trastuzumab plus LY 411575 GSI treatment showed decreased expression of HEY1 and Deltex 1 transcripts compared with trastuzumab alone (Figure 3A). These results would indicate that the lack or delay of breast tumour recurrence observed for the combination of trastuzumab plus GSI treatment could be because of the prevention of the trastuzumabinduced increase in Notch signalling.

\section{Tumour histology, proliferative, and apoptotic characteristics of ErbB-2-positive breast tumour xenografts}

Haematoxylin and eosin staining of tumours excised at week 12, which is prior to trastuzumab-induced regression from Figure 1B, showed that tumours treated with vehicle, trastuzumab, or MRK-003 GSI alone appeared similar in histology (Figure 4A, upper panel). However, tumours treated with trastuzumab plus MRK-003 GSI contained vast numbers of pyknotic nuclei, a possible indication of cell death (Figure 4A, upper panel). Ki67 staining of tumours was used to detect proliferation. The vehicle control, trastuzumab, or MRK-003 GSI-alone treatments had similar numbers of Ki67-positive nuclei (Figure 4A, middle panel) that were quantified using at least 3-5 tumours per group and 60 HPFs (Figure 4B). In contrast, Ki67 staining was almost undetectable in trastuzumab plus MRK-003 GSItreated tumours (Figure 4A, middle panel, and 4B). Furthermore, apoptosis as measured by TUNEL assay showed a $40 \%$ increase in TUNEL-positive cells from trastuzumab plus MRK-003 GSI-treated tumours, consistent with nuclear pyknosis observed by $\mathrm{H} / \mathrm{E}$ (Figure 4A, lower panel, and 4B). These results indicate that the lack of tumour recurrence from trastuzumab plus MRK-003 GSI treatment is probably because of simultaneous induction of apoptosis and downregulation of proliferation. 


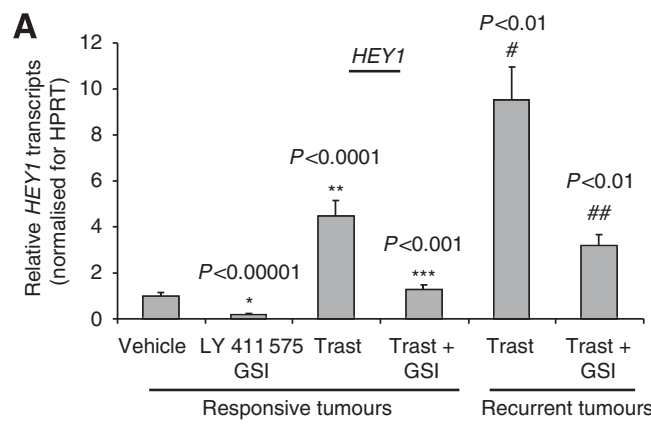

B
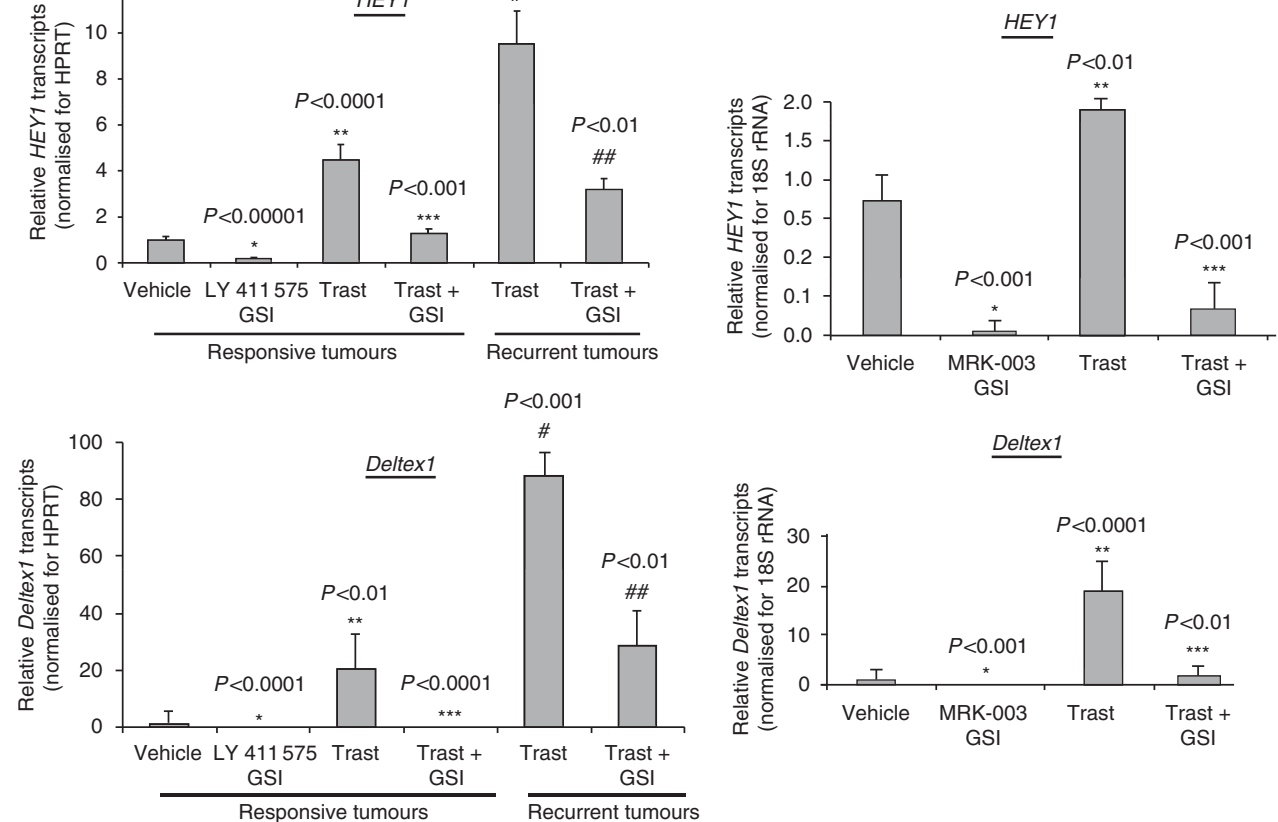

Figure 3 Activity of the Notch-I signalling pathway in BT474 tumours. (A and B) In a separate experiment, I mg of snap-frozen tumours were homogenised and lysed in Trizol reagent and total RNA extracted using the Trizol protocol as described previously (Osipo et al, 2008). Total RNA was reverse transcribed to total cDNA using the Applied Biosystems kit. Real-time PCR was performed using human-specific primers to detect transcripts from Notch target genes: human HEYI and human Deltex I. Human-specific I8S rRNA was detected for normalisation. Results are mean relative transcripts levels compared with vehicle (Control) after normalisation to I8S rRNA. Error bars are s.d. of the mean for five independent tumour samples. *Statistically significant differences between MRK-003 or LY 4II 575 GSI and vehicle (Control). ***tatistically significant differences between trastuzumab (Trast) and Control. *** Statistically significant differences between GSI + Trast and Trast alone. \# Statistically significant differences between Trast-treated tumours and recurrent tumours previously treated with Trast. ${ }^{\# \#}$ Statistically significant differences between GSI + Trast and Trast alone in recurrent tumours.

\section{Expression of activated ErbB-2, ERK1/2, and AKT1 proteins}

The ERK and AKT pathway are activated downstream of overexpressed ErbB-2 (Yarden, 2001; Yarden and Sliwkowski, 2001). Thus, we examined the status of these pathways in treated tumours. BT474 tumours excised at week 12 from Figure 1B were lysed and analysed by western blotting to detect tyrosinephosphorylated ErbB-2 (PY1248), total ErbB-2, phosphorylated ERK1/2, total ERK1/2, phosphorylated AKT1, total AKT1, and PTEN proteins. Figure 4C demonstrates that MRK-003 GSI treatment of BT474 tumours increased PY-ErbB-2 protein compared with vehicle control. Trastuzumab alone showed an $80 \%$ decrease in PY-ErbB-2 protein $v s$ vehicle control (Figure 4C). Interestingly, PY-ErbB-2 protein was reduced $60 \%$ with trastuzumab plus MRK-003 GSI compared with vehicle control (Figure 4C). Furthermore, although either MRK-003 GSI or trastuzumab alone decreased P-ERK1/2 and P-AKT1 compared with vehicle, only trastuzumab plus MRK-003 GSI decreased both P-ERK1/2 and P-AKT1 to almost undetectable levels (Figure 4C). This decrease in P-AKT1 was associated with increased PTEN protein levels (Figure 4C). These results would suggest that downregulation of proliferation and induction of apoptosis by trastuzumab plus MRK-003 GSI could be due at least in part to synergistic or additive inhibition of ERK1/2 and AKT1 activities, which are critical signalling pathways for proliferation and antiapoptosis, respectively.

\section{Antitumour activity of lapatinib, MRK-003 GSI, or combination in ErbB-2-positive breast tumours in vivo}

BT474 breast cancer cells contain ErbB-2 gene amplifications and are sensitive to lapatinib. Breast tumour xenografts were generated using BT474 lapatinib-sensitive cells in athymic nude mice. Growth of tumours was measured in response to vehicle, lapatinib, MRK-003 GSI, or lapatinib plus GSI. The results showed that lapatinib treatment decreased tumour growth by only $40 \%$ compared with the vehicle control (Figure 5A). Treatment of tumours using MRK-003 GSI alone had no statistically significant effect on tumour growth compared with the vehicle control (Figure 5A). However, lapatinib plus GSI showed significant reduction in tumour growth at week 13 compared with GSI alone, lapatinib alone, or vehicle (Figure 5A). The study was stopped at week 13 because of onset of diarrhoea in lapatinib-treated mice.

\section{Tumour histology, proliferative, and apoptotic characteristics of ErbB-2-positive breast tumour xenografts}

Haematoxylin and eosin staining of tumours excised (Figure 5A) at week 10 showed that tumours treated with vehicle, lapatinib, MRK-003 GSI or lapatinib plus GSI appeared similar in histology and confirmed the presence of tumour in the samples excised (Figure 5B, upper panel). Ki67 staining of tumours was used to detect proliferation. The lapatinib, MRK-003 GSI, or lapatinib plus GSI treatments demonstrated $75-90 \%$ reduction in the Ki67positive nuclei compared with the vehicle control (Figure $5 \mathrm{~B}$, middle panel, and $5 \mathrm{C}$ ). Furthermore, apoptosis as measured by TUNEL assay showed a 10-fold increase in TUNEL-positive cells from lapatinib plus MRK-003 GSI-treated tumours compared with vehicle (Figure 5B, third panel, and $5 \mathrm{C}$ ). These results indicate that induction of tumour regression from lapatinib plus MRK-003 GSI treatment is probably also because of simultaneous induction of apoptosis and downregulation of proliferation. 
A

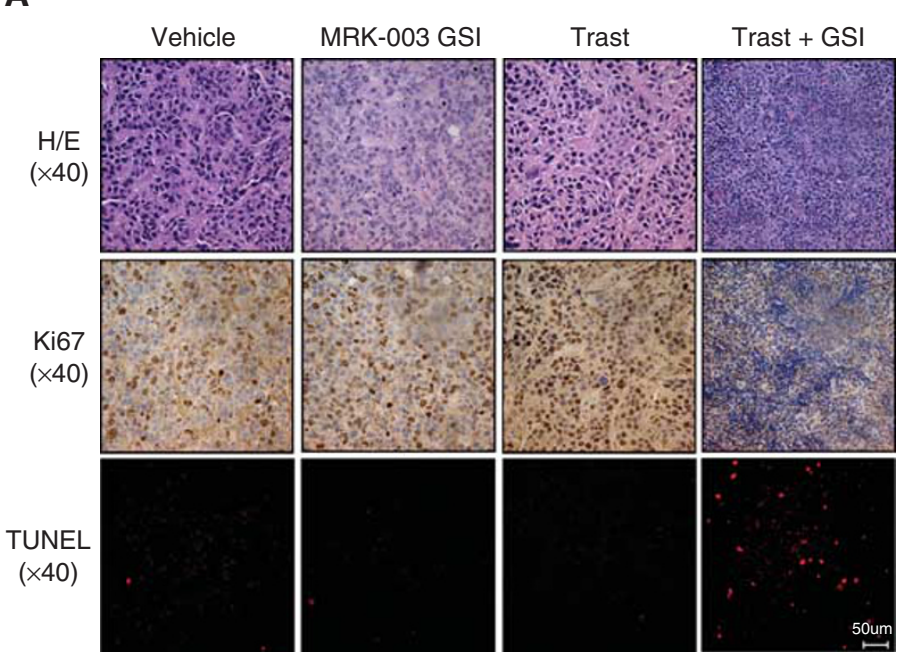

C MRK-003 Trast +

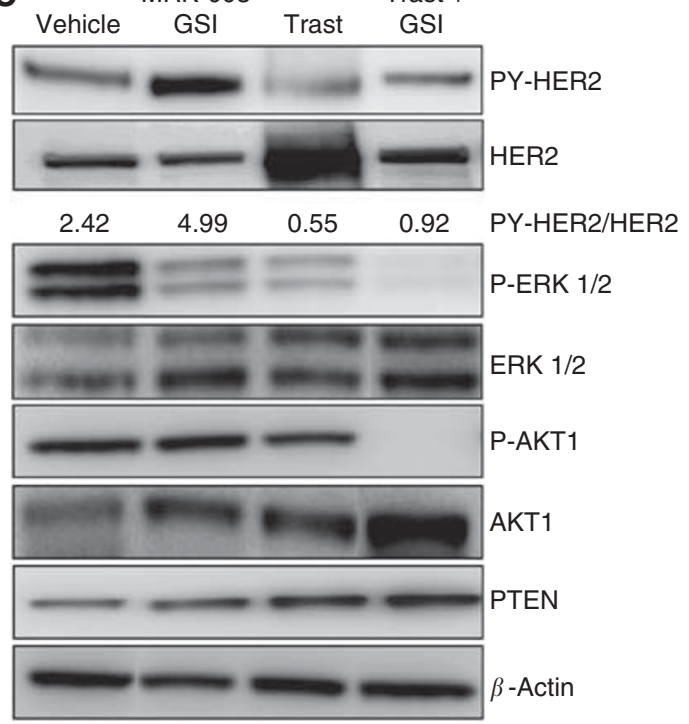

B

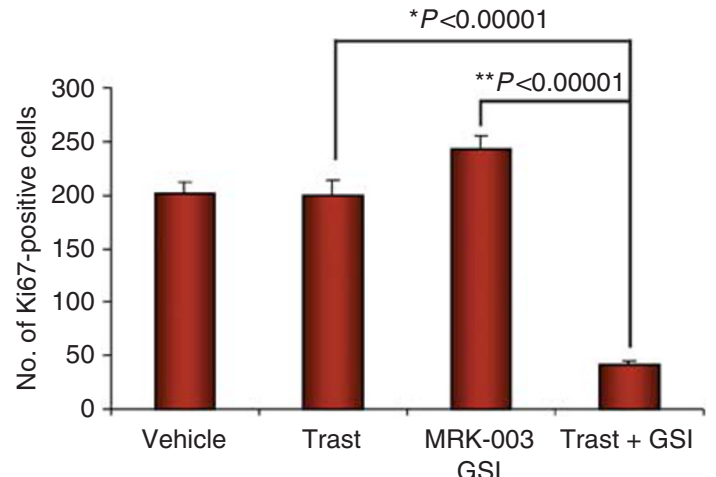

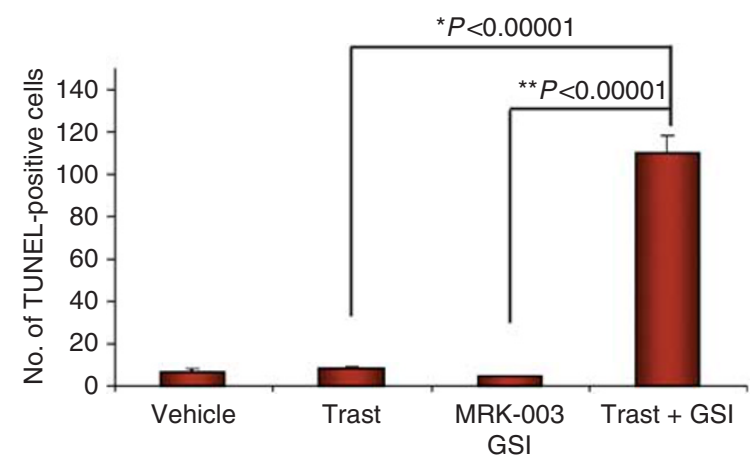

Figure 4 BT474 xenograft histology and signalling pathways. Five mice were euthanised and tumours excised at week I2 as shown in Figure IB. One half of the tumours were immediately fixed in formalin and the remaining half snap-frozen in liquid nitrogen for future study. Fixed tumours were paraffin embedded and sectioned for H/E staining (upper panel), Ki67 (middle panel), and TUNEL (lower panel) assays. All sections were photographed at $\times 40$ magnification using a light microscope. Four panels are shown: BT474 tumours treated with vehicle, trastuzumab (Trast), MRK-003 GSI, or Trast plus GSI. Shown are representative photographs based on at least three tumours. (B) Quantification of Ki67- and TUNEL-positive cells of three tumours using 60 high-powered fields (HPFs) $\times$ at 40 magnification. The $y$ axis represents the number of Ki67- or TUNEL-positive cells for 60 HPFs. The bar graphs are mean \pm s.d.. *Statistical significance between trastuzumab and trastuzumab plus MRK-003 GSI (Trast + MRK-003). **Statistical significance between MRK003 GSI and Trast + MRK-003 GSI. (C) Expression of ErbB-2 and downstream signalling pathways in BT474 tumours. Bits of tumours (I mg) that were snap-frozen in liquid nitrogen were homogenised and lysed in RIPA buffer containing protease and phosphatase inhibitors. Cellular debris was removed by centrifugation at $1000 \mathrm{~g}$ for $5 \mathrm{~min}$ at $4^{\circ} \mathrm{C}$. Supernatants were collected and $25 \mu \mathrm{g}$ of total protein loaded onto a $7 \%$ SDS-PAGE gel followed by western blotting to detect tyrosine phosphorylated ErbB-2 or HER2 (PYI248-HER2), total HER2, P-ERKI/2, total ERKI/2, P-AKTI, total AKTI, PTEN, and actin proteins. The western blot shown is a representative of three independent tumour samples with similar results. Density of bands corresponding to PY-HER2 and total HER2 for each treatment groups was quantified using ImageJ program (NIH, Bethesda, MD, USA) and expressed as a PY-HER2/HER2 ratio of area of the peak.

\section{Expression of activated ErbB-2, ERK1/2, and AKT1 proteins}

BT474 tumours excised (Figure 5A) were lysed and protein extracts were analysed by western blotting to detect tyrosine phosphorylated ErbB-2 (PY1248), total ErbB-2, phosphorylated ERK1/2, total ERK1/2, phosphorylated AKT1 and total AKT1 proteins. Figure 5D demonstrates that lapatinib or MRK-003 GSI treatment alone decreased PY-ErbB-2 protein $40 \%$ compared with vehicle control. Interestingly, lapatinib plus GSI treatment, unlike trastuzumab, had little effect on PY-ErbB-2 protein. However, both phosphorylated ERK-1/2 and AKT1 proteins were reduced in tumours treated with lapatinib plus GSI compared with all other treatments (Figure 5D). These results indicate that induction of tumour regression by lapatinib plus MRK-003 GSI could also be because of simultaneous inhibition of both ERK1/2 and AKT1 activities.

\section{Growth of trastuzumab-resistant BT474 tumours in vivo}

Trastuzumab-resistant, ErbB-2-positive BT474 breast cancer cells were generated by treating cells with $10 \mu \mathrm{g} \mathrm{ml}^{-1}$ trastuzumab for 6 months in vitro as described previously (Osipo et al, 2008). These resistant cells were injected into athymic, nude mice to generate trastuzumab-resistant breast tumour xenografts in vivo. Figure 6A and $\mathrm{B}$ show that xenograft tumours generated from trastuzumabresistant cells were resistant to trastuzumab in two independent studies. Treatment with LY 411575 (Figure 6A) or MRK-003 (Figure 6B) GSI alone did not inhibit tumour growth when compared with vehicle or trastuzumab alone (Figure 6A and $\mathrm{B}$ ). However, trastuzumab plus LY 411575 or MRK-003 GSI decreased the rate of tumour growth by almost $50 \%$ compared with GSI or trastuzumab alone (Figure 6A and B). Results from Figure 6A show significant reduction of tumour growth by trastuzumab plus LY 411575 GSI. However, although results from Figure 6B demon- 
strate reduced tumour growth for trastuzumab plus MRK-003 GSI, this result did not reach statistical significance.

These data taken together suggest that the benefit of using a combination of trastuzumab plus GSI is prevention of tumour recurrence. However, once trastuzumab resistance occurs, a GSI can only partially restore trastuzumab sensitivity.

\section{DISCUSSION}

ErbB-2-positive breast cancer is currently treated with therapeutic agents trastuzumab (Carter et al, 1992) and lapatinib (Nahta et al, 2007), or current preclinical studies (Rimawi et al, 2011) and clinical trials are investigating the combination of trastuzumab plus lapatinib (Baselga et al, 2010). Although trastuzumab plus

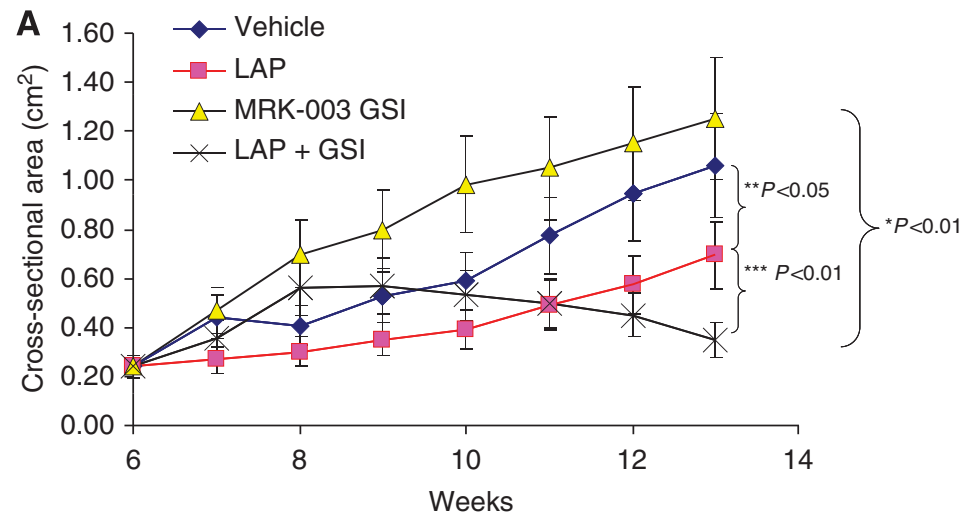

B

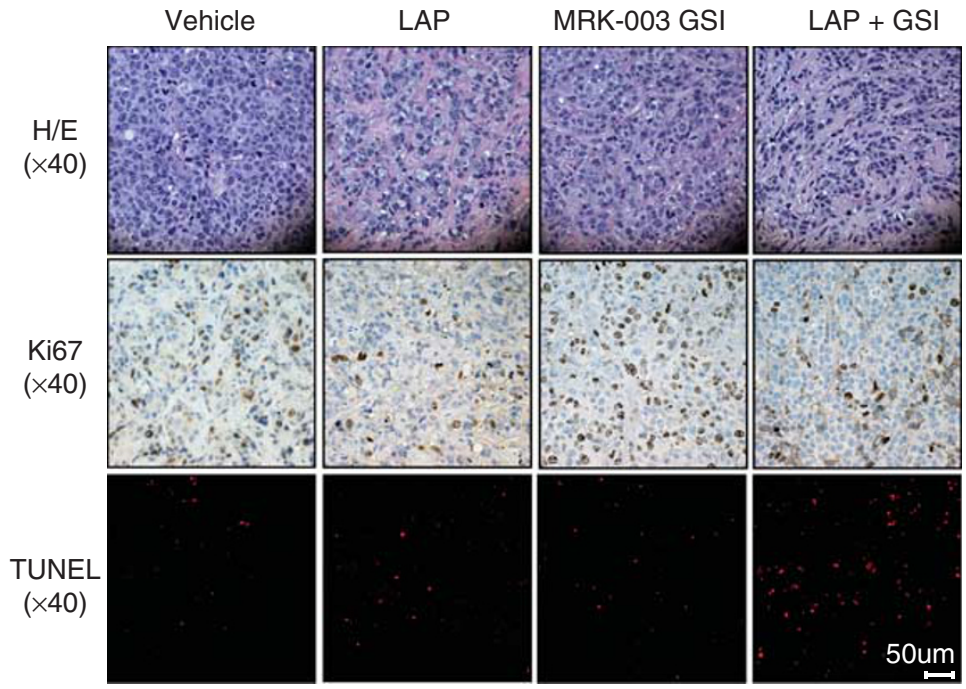

D

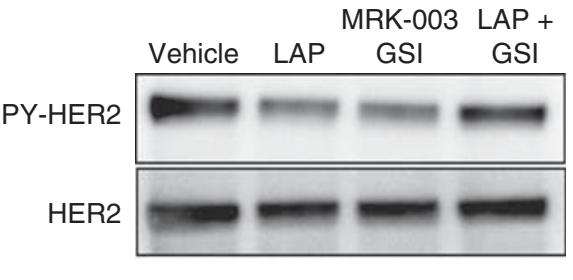

\section{PY-HER2/HER2}
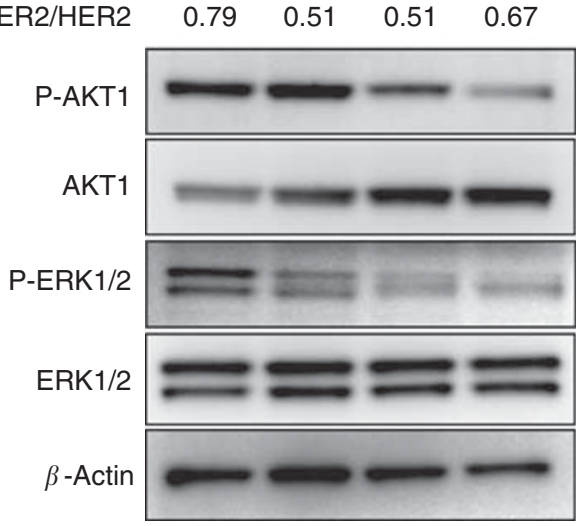

C

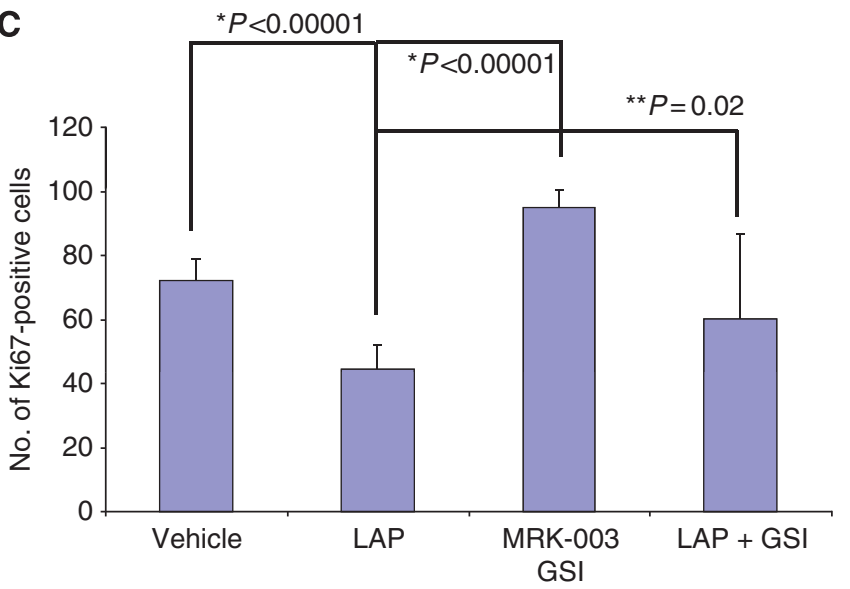

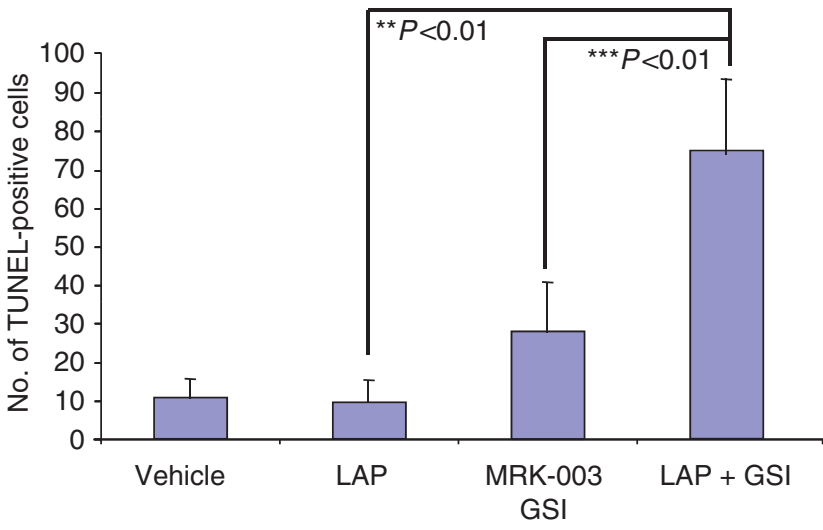

(C) 201I Cancer Research UK 
chemotherapy has been successful in the treatment of ErbB-2positive breast cancer, some patients will not respond to this drug and, among responders, $15 \%$ will have disease recurrence and ultimately progression (Cobleigh et al, 1999; Vogel et al, 2002). Thus, trastuzumab resistance remains a serious clinical problem. One possible reason for this problem could be alterations in signalling pathways that are downstream or parallel to ErbB-2 upon trastuzumab or lapatinib treatment. We showed that ErbB-2 inhibition activates Notch-1 that results in a compensatory
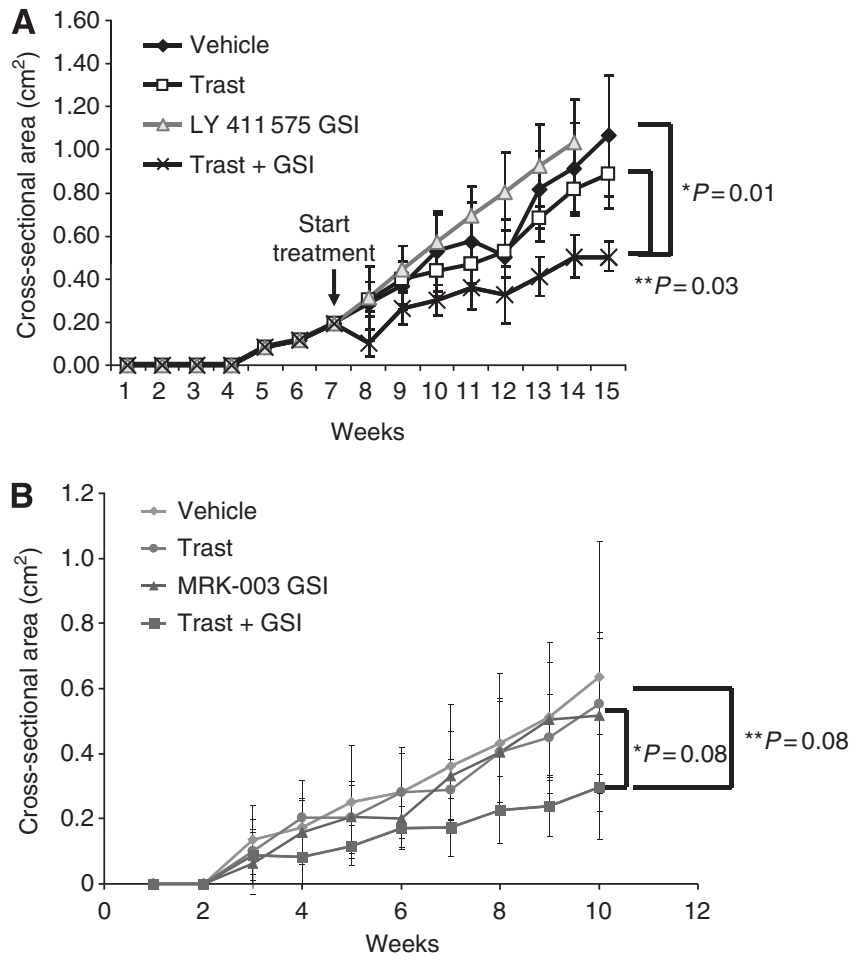

Figure $6 \mathrm{GSI}$ partially restores trastuzumab sensitivity in resistant tumours. (A and $\mathbf{B}$ ) The exact same protocol as described in Figure I was used to generate trastuzumab-resistant tumours in athymic, nude mice using BT474 trastuzumab-resistant cells. (A) LY 4 I I 575 GSI was used. (B) MRK-003 GSI was used. Tumour area (length $\times$ width) was measured weekly using Vernier calipers. The measurements were performed up to I 5 or 10 weeks, respectively. Results show mean tumour cross-sectional area $(($ area $\times \Pi) / 4)$ on the $y$ axis and time in weeks on the $x$ axis. Error bars are s.d. of the mean for 10 mice bearing tumours. *Statistically significant differences between GSI and Trast + GSI. **Statistically significant differences between Trast and Trast + GSI. increase in Notch-1-mediated proliferation (Osipo et al, 2008). Moreover, high ErbB-2 expression in tumour-initiating cells of ErbB-2-positive breast cancer cell lines coincides with high Notch-1 expression and activity (Magnifico et al, 2009). Interestingly, increased ErbB-2 expression in tumour-initiating cells was shown to be Notch-1 dependent (Magnifico et al, 2009). Trastuzumab treatment was able to effectively target tumourinitiating cells of ErbB-2-positive breast cancer cell lines (Magnifico et al, 2009). However, Notch-1 has been implicated in not only the self-renewal of these tumour-initiating cells (Magnifico et al, 2009; Mine et al, 2009; Harrison et al, 2010), but also in trastuzumab resistance (Osipo et al, 2008; Huober et al, 2010). To circumvent these problems, we designed and evaluated for the first time a combination therapeutic strategy that can prevent and/or reverse trastuzumab resistance in vivo. Our data provide, to our knowledge, the first preclinical proof of concept in mice for future clinical trials of combination regimens including trastuzumab and a Notch inhibitor, MRK-003 GSI, for the prevention of tumour recurrence in ErbB-2-positive breast cancer.

Notch-1, a breast oncogene, is a modulator of cell-fate decisions (Politi et al, 2004). Overexpression of constitutively active forms of Notch-1, Notch-3, and Notch-4 develop spontaneous murine mammary tumours in vivo (Callahan and Raafat, 2001). Notch-4 has been shown to be critical for the survival of tumour-initiating cells (Magnifico et al, 2009; Mine et al, 2009; Harrison et al, 2010). In addition, Notch-1 has been recently suggested as a novel marker of trastuzumab resistance from human breast cancer tissue (Huober et al, 2010). We have identified Notch-1 as a novel target in trastuzumab-resistant breast cancer in vitro (Osipo et al, 2008). Our findings, along with evidence from the literature, indicate that Notch could be an important target in trastuzumab-resistant, ErbB-2-positive breast cancer. One class of compounds that are being used to inhibit the Notch pathway are GSIs that are currently in clinical trials for the treatment of breast cancer and other solid tumours (Pannuti et al, 2010). Recently, it was reported that MRK003 GSI treatment of Balb/c-neuT female mice reduced tumour onset, tumour burden, and AKT1/mTOR activities associated with ErbB-2-positive, murine breast tumours (Efferson et al, 2010).

Our results demonstrate that although trastuzumab treatment caused virtually complete tumour regression (Figure 1A and B), which mimics what is observed in the clinic, it could not prevent tumour recurrence in $40 \%$ of the mice (Figure $1 \mathrm{~A}$ and $\mathrm{B}$, and 2). However, adding a GSI to trastuzumab treatment either completely abolished (MRK-003) or significantly reduced tumour recurrence (Figure $1 \mathrm{~A}$ and $\mathrm{B}$, and 2). Interestingly, recurrent tumours posttrastuzumab plus LY 411575 GSI treatment showed a significant decrease in Notch transcriptional activity compared with trastuzumab treatment alone, suggesting that Notch signalling could be responsible for ErbB-2-positive breast tumour recurrence post-

Figure 5 Lapatinib plus MRK-003 GSI reduces tumour growth. (A) ErbB-2-overexpressing BT474 xenografts were generated in 40 ovariectomised, athymic nude mice by injecting $5 \times 10^{6}$ cells into both mammary fat pads. Once tumours reached a mean tumour cross-sectional area of $0.25 \mathrm{~cm}^{2}$, mice were randomised and treated with vehicle, lapatinib (LAP), MRK-003 GSI, or LAP plus GSI. Tumour area (length $\times$ width) was measured weekly using Vernier calipers. The measurements were performed up to 13 weeks. Results show mean tumour cross-sectional area $((\operatorname{area} \times \Pi) / 4)$ on the $y$ axis and time in weeks on the $x$ axis. Error bars are s.d. of the mean for 10 mice bearing tumours. (B) Fixed tumours were paraffin embedded and sectioned for $H / E$ staining (upper panel), Ki67 (middle panel), and TUNEL (lower panel) assays. All sections were photographed at $\times 40$ using a light microscope. Four panels are shown: BT474 tumours treated with vehicle, lapatinib (LAP), MRK-003 GSI, and LAP + GSI. The photographs of a single tumour sample are representative of three tumours with similar results. (C) Quantification of Ki67- and TUNEL-positive cells of three tumours using 60 high-powered fields (HPFs) at $\times 40$ magnification. The $y$ axis represents the number of Ki67- or TUNEL-positive cells per $60 \mathrm{HPFs}$. The bar graphs are mean \pm s.d. *Statistical differences compared with vehicle control. *** Statistical differences between LAP and LAP + GSI. ****Statistical difference between MRK-003 GSI and LAP + GSI. Statistical analysis was performed using a two-sided, nonpaired Student's t-test. (D) Bits of tumours (I mg) that were snap-frozen in liquid nitrogen were homogenised and lysed in RIPA buffer containing protease and phosphatase inhibitors. Cellular debris was removed by centrifugation at $1000 \mathrm{~g}$ for $5 \mathrm{~min}$ at $4^{\circ} \mathrm{C}$. Supernatants were collected and $25 \mu \mathrm{g}$ of total protein loaded onto a 7\% SDS-PAGE gel followed by western blotting to detect tyrosine phosphorylated ErbB-2 or HER2 (PY I 248-HER2), total HER2, P-ERKI/2, total ERKI/2, P-AKTI, total AKTI, and actin proteins. Densitometry was performed on PY-HER2 and total HER2 bands for each treatment groups using Imagel program and expressed as a PY-HER2/HER2 ratio of area of the peak. Western blotting was performed on at least three independent tumour samples. Representative western blots are shown with similar results. *Statistically significant differences between mean slopes of the curve for LAP plus GSI and GSI alone. **Statistically significant differences between mean slopes of the curve for LAP and vehicle control. ****Statistically significant differences between mean slopes of the curve for LAP plus GSI and LAP alone. Linear regression analyses were performed for tumour growth curve. 
trastuzumab treatment. Consistent with the literature, our data could also suggest that Notch plays a critical role in the survival of tumour-initiating cells as demonstrated by the lack of tumour recurrence when Notch was inhibited in combination with ErbB-2 inhibition. Although the data presented here suggest a role for Notch based on Notch target genes modulated by a GSI, the effects on tumour recurrence could alternatively be the result of a $\gamma$-secretase-mediated effect as it is known that a GSI specifically inhibits $\gamma$-secretase activity.

Treatment with the combination of trastuzumab plus MRK-003 GSI simultaneously decreased proliferation and induced tumour cell death (Figure 4). These antitumour effects of the combination therapy may be because of near-complete blockade of two critical signalling pathways downstream of ErbB-2: ERK1/2 and AKT1 (Figure 4). Thus, a combination of trastuzumab plus MRK-003 GSI could benefit those women with recurrent, or possibly resistant, ErbB-2-positive breast cancer ultimately to reduce or eliminate disease progression and deaths by simultaneously inactivating two critical prosurvival and antiapoptotic pathways such as ERK1/2 and AKT1. The exact mechanism by which the combination treatment inhibits ERK1/2 and AKT1 activities is currently under investigation.

Lapatinib is a very potent inhibitor of ErbB-2 activity in vitro; however, lapatinib alone reduced tumour growth by only $40 \%$ in our BT474 model of ErbB-2-positive breast cancer (Figure 5A). A combination of lapatinib plus MRK-003 GSI showed significant reduction in the tumour growth (Figure $5 \mathrm{~A}$ ). This is likely because of inhibition of ERK1/2 and AKT1 activities (Figure 5D) that resulted in increased apoptosis and decreased proliferation (Figure $5 \mathrm{~B}$ and $\mathrm{C}$ ). However, the onset of diarrhoea-associated toxicity with lapatinib or lapatinib plus GSI treatment at week 13 caused the study to end prematurely and, therefore, complete tumour regression was not reached.

Our results also showed that a GSI could partially restore sensitivity to trastuzumab in resistant tumours (Figure 6). The mechanism by which a GSI only partially reverses trastuzumab resistance in vivo is being actively studied. It is possible that targeting all four Notch receptors with a pan-Notch inhibitor such as a GSI might not effectively target Notch-1, which we have shown to be necessary for trastuzumab resistance in vitro (Osipo et al, 2008). A more specific Notch-1 or possibly other Notch signalling pathway inhibitors could prove to be more effective and potent. We are currently investigating which components of Notch signalling should be targeted in trastuzumab- or lapatinib-resistant tumours to more effectively induce tumour regression. Therapeutic targeting of Notch receptors using antibodies could prove to be a more potent and specific mode of inhibition (Wu et al, 2010) and has not been fully investigated in breast cancer. Furthermore, the Notch pathway is complicated in tumours because of multiple modes of action. For example, it is known that Delta-like 4 on endothelial cells engages and activates the Notch-1 receptor on cancer epithelial cells to promote angiogenesis (Yan et al, 2010). In addition, Notch signalling has been recently implicated to play a role in survival and differentiation of tumour stroma (Orr et al, 2009). The level of complexity for the role of Notch signalling in the tumour microenvironment requires a thorough investigation of the Notch pathway in breast cancer, and most notably in antiErbB-2-targeted drug resistance, with the goal of identifying novel and specific targets to treat or reverse resistance.

We investigated the therapeutic benefits of two distinct inhibitors to block ErbB-2 (trastuzumab or lapatinib) and Notch (MRK-003 or LY 411575 GSI) pathways. Although lapatinib is a more potent inhibitor of ErbB-2 activity in vitro compared with trastuzumab, trastuzumab is more efficient in inhibiting BT474 tumour growth. As the mice used in this study are immunodeficient, this is not likely to be an immune-mediated effect (e.g., ADCC). Lapatinib plus MRK-003 GSI were not as effective as trastuzumab plus MRK-003 GSI in inhibiting tumour growth of BT474 xenografts. A combination of MRK-003 GSI plus trastuzumab was sufficient to prevent tumour recurrence, whereas a combination of LY 411575 plus trastuzumab was only able to reduce tumour recurrence. The observed differences in efficacy between MRK-003 and LY 411575 GSI was also observed by recent data from Grudzien et al (2010), where the effect of MRK-003 was irreversible, leading to complete elimination of tumour-initiating cells in vitro, whereas the effect of LY 411575 was reversible after drug washout. These results, in addition to data from the current paper, suggest that the best combination strategy for a future clinical trial to prevent tumour recurrence of ErbB-2-positive breast cancer could be trastuzumab plus MRK-003 GSI.

In conclusion, our findings suggest for the first time that the benefit of using a combination of trastuzumab plus a GSI is prevention of ErbB-2-positive breast tumour recurrence. Because Notch is a breast oncogene that is critical for survival and proliferation of breast cancer cells, our findings strongly suggest that combined treatment with a Notch inhibitor should be an effective therapeutic strategy to prevent tumour recurrence and possibly disease progression and death in ErbB-2-positive breast cancer. Our data also suggest that a combination of trastuzumab plus MRK-003 GSI could benefit women with recurrent, or possibly resistant, ErbB-2-positive breast cancer to prevent disease progression.

\section{ACKNOWLEDGEMENTS}

We are grateful to Drs Maurizio Bocchetta, Paola Rizzo, and Mitchell Denning for helpful advice and commentaries during this work. We are grateful for the Department of Defense Idea Award (W81XWH-08-1-0643/BC074462 to C Osipo) for financially supporting portions of this study.

\section{Conflict of interest}

The authors declare no conflict of interest.

\section{REFERENCES}

Amar S, Moreno-Aspitia A, Perez EA (2008) Issues and controversies in the treatment of HER2 positive metastatic breast cancer. Breast Cancer Res Treat 109: $1-7$

Baselga J, Albanell J, Molina MA, Arribas J (2001) Mechanism of action of trastuzumab and scientific update. Semin Oncol 28: 4-11

Baselga J, Bradbury I, Eidmann H, Di Cosimo S, Aura C, De Azabuja E, Gomez H, Dinh P, Fauria K, Van Dooren V, Paoletti P, Goldhirsch A, Chang T-W, Lang I, Unitch M, Gelber RD, Piccart-Gebhart M (2010) First results of the NeoALTO trial (BIG 01- 06/EGF 106903): a phase III, randomized, open label, neoadjuvant study of lapatinib, trastuzumab, and their combination plus paclitaxel in women with HER- 2 positive primary breast cancer. Cancer Res (Supplemental) 70: 1s-490s
Benz CC, Scott GK, Sarup JC, Johnson RM, Tripathy D, Coronado E, Shepard HM, Osborne CK (1992) Estrogen-dependent, tamoxifenresistant tumorigenic growth of MCF-7 cells transfected with HER2/ neu. Breast Cancer Res Treat 24: 85-95

Bettenhausen B, Hrabe de Angelis M, Simon D, Guenet JL, Gossler A (1995) Transient and restricted expression during mouse embryogenesis of Dll1, a murine gene closely related to Drosophila Delta. Development 121: 2407-2418

Blaumueller CM, Qi H, Zagouras P, Artavanis-Tsakonas S (1997) Intracellular cleavage of Notch leads to a heterodimeric receptor on the plasma membrane. Cell 90: 281-291

Brodie A, Macedo L, Sabnis G (2009) Aromatase resistance mechanisms in model systems in vivo. J Steroid Biochem Mol Biol 118: 283-287 
Callahan R, Raafat A (2001) Notch signaling in mammary gland tumorigenesis. J Mammary Gland Biol Neoplasia 6: 23-36

Carter P, Presta L, Gorman CM, Ridgway JB, Henner D, Wong WL, Rowland AM, Kotts C, Carver ME, Shepard HM (1992) Humanization of an anti-p185HER2 antibody for human cancer therapy. Proc Natl Acad Sci USA 89: $4285-4289$

Cobleigh MA, Vogel CL, Tripathy D, Robert NJ, Scholl S, Fehrenbacher L, Wolter JM, Paton V, Shak S, Lieberman G, Slamon DJ (1999) Multinational study of the efficacy and safety of humanized anti-HER2 monoclonal antibody in women who have HER2- overexpressing metastatic breast cancer that has progressed after chemotherapy for metastatic disease. J Clin Oncol 17: 2639-2648

Dickson BC, Mulligan AM, Zhang H, Lockwood G, O'Malley FP, Egan SE, Reedijk M (2007) High-level JAG1 mRNA and protein predict poor outcome in breast cancer. Mod Pathol 20: 685-693

Dievart A, Beaulieu N, Jolicoeur P (1999) Involvement of Notch1 in the development of mouse mammary tumors. Oncogene 18: 5973-5981

Dunwoodie SL, Henrique D, Harrison SM, Beddington RS (1997) Mouse Dll3: a novel divergent Delta gene which may complement the function of other Delta homologues during early pattern formation in the mouse embryo. Development 124: 3065-3076

Efferson CL, Winkelmann CT, Ware C, Sullivan T, Giampaoli S, Tammam J, Patel S, Mesiti G, Reilly JF, Gibson RE, Buser C, Yeatman T, Coppola D, Winter C, Clark EA, Draetta GF, Strack PR, Majumder PK (2010) Downregulation of notch pathway by a \{gamma\}-secretase inhibitor attenuates AKT/mammalian target of rapamycin signaling and glucose uptake in an ERBB2 transgenic breast cancer model. Cancer Res 70: $2476-2484$

Grudzien P, Lo S, Albain KS, Robinson P, Rajan P, Strack PR, Golde TE, Miele L, Foreman KE (2010) Inhibition of Notch signaling reduces the stem-like population of breast cancer cells and prevents mammosphere formation. Anticancer Res 30: 3853-3867

Harrison H, Farnie G, Howell SJ, Rock RE, Stylianou S, Brennan KR, Bundred NJ, Clarke RB (2010) Regulation of breast cancer stem cell activity by signaling through the Notch4 receptor. Cancer Res 70: $709-718$

Huober J, Loibl S, Darb-Esfahani S, Solbach C, Tesch H, Holms F, Fehm T, von Minckwitz G, Mehta K, Denkert C (2010) New molecular biomarkers for resistance to trastuzumab in primary HER2 positive breast cancer - a translational investigation from the Neoadjuvant GeparQuattro Study. Cancer Res 70(24 Suppl): 100s

Kaufman B, Trudeau M, Awada A, Blackwell K, Bachelot T, Salazar V, DeSilvio M, Westlund R, Zaks T, Spector N, Johnston S (2009) Lapatinib monotherapy in patients with HER2-overexpressing relapsed or refractory inflammatory breast cancer: final results and survival of the expanded HER2+ cohort in EGF103009, a phase II study. Lancet Oncol 10: $581-588$

Kopan R, Ilagan MX (2009) The canonical Notch signaling pathway: unfolding the activation mechanism. Cell 137: 216-233

Lacerda L, Pusztai L, Woodward WA (2010) The role of tumor initiating cells in drug resistance of breast cancer: implications for future therapeutic approaches. Drug Resist Updat 13: 99-108

Li X, Lewis MT, Huang J, Gutierrez C, Osborne CK, Wu MF, Hilsenbeck SG, Pavlick A, Zhang X, Chamness GC, Wong H, Rosen J, Chang JC (2008) Intrinsic resistance of tumorigenic breast cancer cells to chemotherapy. $J$ Natl Cancer Inst 100: 672-679

Lindsell CE, Shawber CJ, Boulter J, Weinmaster G (1995) Jagged: a mammalian ligand that activates Notch1. Cell 80: 909-917

Logeat F, Bessia C, Brou C, LeBail O, Jarriault S, Seidah NG, Israel A (1998) The Notch1 receptor is cleaved constitutively by a furin-like convertase. Proc Natl Acad Sci USA 95: 8108-8112

Magnifico A, Albano L, Campaner S, Delia D, Castiglioni F, Gasparini P, Sozzi G, Fontanella E, Menard S, Tagliabue E (2009) Tumor-initiating cells of HER2-positive carcinoma cell lines express the highest oncoprotein levels and are sensitive to trastuzumab. Clin Cancer Res 15: 2010-2021

Menendez JA, Mehmi I, Lupu R (2006) Trastuzumab in combination with heregulin-activated Her-2 (erbB-2) triggers a receptor-enhanced chemosensitivity effect in the absence of Her-2 overexpression. J Clin Oncol 24: $3735-3746$

Mine T, Matsueda S, Li Y, Tokumitsu H, Gao H, Danes C, Wong KK, Wang X, Ferrone S, Ioannides CG (2009) Breast cancer cells expressing stem cell markers CD44+ CD24 lo are eliminated by Numb-1 peptideactivated T cells. Cancer Immunol Immunother 58: 1185-1194

Muss HB, Thor AD, Berry DA, Kute T, Liu ET, Koerner F, Cirrincione CT, Budman DR, Wood WC, Barcos M, Henderson IC (1994) c-erbB-2 expression and response to adjuvant therapy in women with nodepositive early breast cancer. $N$ Engl J Med 330: 1260-1266

Nabholtz JM, Slamon D (2001) New adjuvant strategies for breast cancer: meeting the challenge of integrating chemotherapy and trastuzumab (Herceptin). Semin Oncol 28: 1-12

Nagata Y, Lan KH, Zhou X, Tan M, Esteva FJ, Sahin AA, Klos KS, Li P, Monia BP, Nguyen NT, Hortobagyi GN, Hung MC, Yu D (2004) PTEN activation contributes to tumor inhibition by trastuzumab, and loss of PTEN predicts trastuzumab resistance in patients. Cancer Cell 6: $117-127$

Nahta R, Esteva FJ (2006) Herceptin: mechanisms of action and resistance. Cancer Lett 232: $123-138$

Nahta R, Yuan LX, Du Y, Esteva FJ (2007) Lapatinib induces apoptosis in trastuzumab-resistant breast cancer cells: effects on insulin-like growth factor I signaling. Mol Cancer Ther 6: 667-674

O'Regan RM, Cisneros A, England GM, MacGregor JI, Muenzner HD, Assikis VJ, Bilimoria MM, Piette M, Dragan YP, Pitot HC, Chatterton R, Jordan VC (1998) Effects of the antiestrogens tamoxifen, toremifene, and ICI 182,780 on endometrial cancer growth. J Natl Cancer Inst 90: $1552-1558$

Orr B, Grace OC, Vanpoucke G, Ashley GR, Thomson AA (2009) A role for notch signaling in stromal survival and differentiation during prostate development. Endocrinology 150: 463-472

Osipo C, Gajdos C, Cheng D, Jordan VC (2005a) Reversal of tamoxifen resistant breast cancer by low dose estrogen therapy. J Steroid Biochem Mol Biol 93: 249-256

Osipo C, Gajdos C, Liu H, Chen B, Jordan VC (2003) Paradoxical action of fulvestrant in estradiol-induced regression of tamoxifen-stimulated breast cancer. J Natl Cancer Inst 95: 1597-1608

Osipo C, Meeke K, Liu H, Cheng D, Lim S, Weichel A, Jordan VC (2005b) Trastuzumab therapy for tamoxifen-stimulated endometrial cancer. Cancer Res 65: 8504-8513

Osipo C, Patel P, Rizzo P, Clementz AG, Hao L, Golde TE, Miele L (2008) ErbB-2 inhibition activates Notch-1 and sensitizes breast cancer cells to a gamma-secretase inhibitor. Oncogene 27: 5019-5032

Pannuti A, Foreman K, Rizzo P, Osipo C, Golde T, Osborne B, Miele L (2010) Targeting Notch to target cancer stem cells. Clin Cancer Res 16: $3141-3152$

Piechocki MP, Yoo GH, Dibbley SK, Lonardo F (2007) Breast cancer expressing the activated HER2/neu is sensitive to gefitinib in vitro and in vivo and acquires resistance through a novel point mutation in the HER2/neu. Cancer Res 67: 6825-6843

Politi K, Feirt N, Kitajewski J (2004) Notch in mammary gland development and breast cancer. Semin Cancer Biol 14: $341-347$

Reedijk M, Odorcic S, Chang L, Zhang H, Miller N, McCready DR, Lockwood G, Egan SE (2005) High-level coexpression of JAG1 and NOTCH1 is observed in human breast cancer and is associated with poor overall survival. Cancer Res 65: 8530-8537

Rimawi MF, Wiechmann LS, Wang YC, Huang C, Migliaccio I, Wu MF, Gutierrez C, Hilsenbeck SG, Arpino G, Massarweh S, Ward R, Soliz RD, Osborne CK, Schiff R (2011) Reduced dose and intermittent treatment with lapatinib and trastuzumab for potent blockade of the HER pathway in HER-2/neu overexpressing breast tumor xenografts. Clin Cancer Res 17: $1351-1361$

Shou J, Massarweh S, Osborne CK, Wakeling AE, Ali S, Weiss H, Schiff R (2004) Mechanisms of tamoxifen resistance: increased estrogen receptorHER2/neu cross-talk in ER/HER2-positive breast cancer. J Natl Cancer Inst 96: $926-935$

Shutter JR, Scully S, Fan W, Richards WG, Kitajewski J, Deblandre GA, Kintner CR, Stark KL (2000) Dll4, a novel Notch ligand expressed in arterial endothelium. Genes Dev 14: 1313-1318

Slamon D, Pegram M (2001) Rationale for trastuzumab (Herceptin) in adjuvant breast cancer trials. Semin Oncol 28: 13-19

Slamon DJ, Godolphin W, Jones LA, Holt JA, Wong SG, Keith DE, Levin WJ, Stuart SG, Udove J, Ullrich A, Press MF (1989) Studies of the HER-2/neu proto-oncogene in human breast and ovarian cancer. Science 244: $707-712$

Sliwkowski MX, Lofgren JA, Lewis GD, Hotaling TE, Fendly BM, Fox JA (1999) Nonclinical studies addressing the mechanism of action of trastuzumab (Herceptin). Semin Oncol 26: 60-70

Vogel CL, Cobleigh MA, Tripathy D, Gutheil JC, Harris LN, Fehrenbacher L, Slamon DJ, Murphy M, Novotny WF, Burchmore M, Shak S, Stewart SJ, Press M (2002) Efficacy and safety of trastuzumab as a single agent in first-line treatment of HER2- overexpressing metastatic breast cancer. J Clin Oncol 20: 719-726 
Widakowich C, Dinh P, de Azambuja E, Awada A, Piccart-Gebhart M (2008) HER-2 positive breast cancer: what else beyond trastuzumabbased therapy? Anticancer Agents Med Chem 8: 488-496

Wu Y, Cain-Hom C, Choy L, Hagenbeek TJ, de Leon GP, Chen Y, Finkle D, Venook R, Wu X, Ridgway J, Schahin-Reed D, Dow GJ, Shelton A, Stawicki S, Watts RJ, Zhang J, Choy R, Howard P, Kadyk L, Yan M, Zha J, Callahan CA, Hymowitz SG, Siebel CW (2010) Therapeutic antibody targeting of individual Notch receptors. Nature 464: $1052-1057$

Xia W, Bacus S, Hegde P, Husain I, Strum J, Liu L, Paulazzo G, Lyass L, Trusk P, Hill J, Harris J, Spector NL (2006) A model of acquired autoresistance to a potent ErbB2 tyrosine kinase inhibitor and a therapeutic strategy to prevent its onset in breast cancer. Proc Natl Acad Sci USA 103: 7795-7800
Yan M, Callahan CA, Beyer JC, Allamneni KP, Zhang G, Ridgway JB, Niessen K, Plowman GD (2010) Chronic DLL4 blockade induces vascular neoplasms. Nature 463: E6-E7

Yao K, Rizzo P, Rajan P, Albain K, Rychlik K, Sha S, Miele L (2010) Notch-1 and Notch-4 receptors as prognostic markers in breast cancer. Int J Surg Pathol; e-pub ahead of print 5 May 2010; doi:10.1177/1066896910362080

Yarden Y (2001) Biology of HER2 and its importance in breast cancer. Oncology 61(Suppl 2): 1-13

Yarden Y, Sliwkowski MX (2001) Untangling the ErbB signalling network. Nat Rev Mol Cell Biol 2: 127-137

Yokoyama G, Fujii T, Ogo E, Yanaga H, Toh U, Yamaguchi M, Mishima M, Takamori S, Shirouzu K, Yamana H (2005) Advanced chemoresistant breast cancer responding to multidisciplinary treatment with hyperthermia, radiotherapy, and intraarterial infusion. Int J Clin Oncol 10: 139-143

This work is published under the standard license to publish agreement. After 12 months the work will become freely available and the license terms will switch to a Creative Commons Attribution-NonCommercial-Share Alike 3.0 Unported License. 